\title{
Post-consumer PET Bottle Recycling: Chemical Dose Optimization
}

\author{
Md. Shahadat Hossain* and Md Anisur Rahman \\ Department of Chemical Engineering and Polymer Science, Shahjalal University of Science and Technology, Sylhet - 3114, \\ Bangladesh
}

Received: August 17, 2020, Revised: September 11, 2020, Accepted: September 12, 2020, Available Online: September 15, 2020

\begin{abstract}
Polyethylene terephthalate (PET) bottles are being used in our daily life and consequently go to the landfills after their use. Additionally, virgin PET resins are produced from nonrenewable resources, such as fossil fuels, whose reserves are depleting continuously. Therefore, to maintain ecological and environmental balances as well as for sustainable development, post-consumer PET (pcrPET) bottles should be recycled. Among many recycling processes, mechanical recycling of pcrPET is attractive due to lower cost involvement. One of the most crucial and important processes of mechanical recycling is hot washing for contaminants removal. Hot washing uses a cleaning solution made of caustic soda $(\mathrm{NaOH})$ and detergent at elevated temperature. In this paper, caustic soda and FORYL LFO (FLO) detergent doses were changed gradually to investigate effective contaminants removal through colorimetric study. Finally, concentration vs. absorbance graphs from colorimetric study suggests that $2 \% \mathrm{NaOH}$ and $2 \%$ FLO detergent is the optimum chemical dose at hot washing for pcrPET recycling.
\end{abstract}

Keywords: PET Bottle; Recycling; Chemical Dose; $\mathrm{NaOH}$; Detergent; Colorimetric Analysis.

This work is licensed under a Creative Commons Attribution-Non Commercial 4.0 International

\section{Introduction}

Plastic bottles are widely used at beverage, water, oil, and pharmaceutical industries in Bangladesh [1], [2]. Most of them are directly goes to the landfill after their first hand use but those bottles are not biodegradable [3]. As a result, they create potential hazard for our agricultural lands, sewage systems as well as for water networks across the country [4]. On the contrary, Bangladesh is currently producing different plastic products from both imported virgin polymer and locally recycled plastics [3], [5]. Bangladesh used 75,000 metric tons (MT) plastic raw materials mainly for packaging materials, household accessories and producing construction materials in 2015 [5], [6]. At the same time, Bangladesh recycled around 65 percent of its post consumer plastic materials and this trend was increasing in every year [2], [6]. This post consumer plastic recycling provides Bangladesh two fold advantages: firstly for plastic waste management and secondly for saving money which otherwise would have been used for importing virgin polymer [4], [6],[7]. Unfortunately, up to now most of the recycled plastic materials are used only by small and medium-sized enterprises (SMEs) and yet no big plastic conglomerates have used recycled plastic materials due to its inferior quality [5]. In a book, United Nations ESCAP [6] showed that SMEs used 60 percent of the total recycled plastic materials and subsequently produced low quality plastic products. Rest of the unused and recycled plastic materials was exported to China. For instance, Bangladesh exported USD 6 million worth recycled plastic materials between July 2008 and January 2010 due to lack of proper recycling technology [5], [6]. However, Bangladesh has huge demand for quality recycled plastic materials inside the country [2]. Therefore, recycled plastic materials quality should be improved through proper plastic collection method and proper washing for decontamination.
Among various plastic materials, most widely used plastics in Bangladesh are polyethylene (PE), polyvinylchloride (PVC), polypropylene (PP), polyethylene terephthalate (PET) and polystyrene (PS) [2], [3]. Among which post consumer PET (pcrPET) is recycled mostly and has been used by SMEs for bottle production for beverage, oil, water, and medicine industry as well as for PET sheet and polyester fiber production. But still now pcrPET is recycled in Bangladesh by conventional method where it is manually sorted firstly, later grounded and finally cold washed by detergent and subsequently dried for decontamination. But, this conventional recycling method produces inferior quality recycled PET flakes in Bangladesh.

However, it is widely known that during pcrPET materials recycling, caustic soda $(\mathrm{NaOH})$ and detergent are used in hot washing, but dosage of chemicals is different in different countries [8]-[10]. This is because pcrPET bottles are collected in wide variety of methods in different counties, which make them contain different amounts of dirt and contaminants [11][13]. On the basis of contaminants adherence to pcrPET bottles after their first hand use, $1-3 \% \mathrm{NaOH}$ along with small amount of detergent are used for contaminants removal [8], [10]. Some authors also report usage of antifoaming agent in hot washing for foaming phenomena reduction [8]. Therefore, if pcrPET bottles are recycled in Bangladesh, chemical dosage in hot washing should be determined by considering amount and types of contaminants present in those post consumer bottles [14], [15]. If improper amount of washing chemicals are used in hot washing step of recycling, it will give yellowish color in subsequent recycling steps, for example, extrusion, injection molding, and blow molding due to presence of trace amount of $\mathrm{NaOH}$ in recycled PET materials [13], [16]-[19]. Therefore, determination of chemical dosage in hot washing is crucial for total pcrPET bottle recycling. Use of proper chemical dosage will 
turn potential environmental hazard into valuable PET flakes and subsequently will reduce the environmental pollution through PET bottles recycling. In this paper, pcrPET bottles generated across the country were collected and then a step by step recycling process was developed for contaminants removal by employing chemical dosage. Different chemical dose - varying proportion of $\mathrm{NaOH}$ and FORYL LFO (FLO) detergent - were tested in hot washing, a crucial step of recycling process, to find out the optimum chemical dose for pcrPET bottles recycling.

\section{Methodology}

\subsection{Experimental Set-up}

It was assumed in this study that a pcrPET recycling plant is running with a production capacity of 1.0 MT recycled PET flakes per hour where 30 minutes of hot washing for pcrPET flakes is employed. A hot washer (is shown in Fig. 1) with a $100 \mathrm{rpm}$ stirrer uses $6 \mathrm{~m}^{3}$ water and $\mathrm{NaOH}$ plus FLO detergent dose for pcrPET flakes hot washing. Therefore, it is estimated that every 30 minutes hot washer contains 0.50 MT flakes within $6 \mathrm{~m}^{3}$ of water for hot washing. However, this paper used small scale hot washer accommodating 1 liter (L) water for 30 minutes of hot washing. Consequently, $0.083 \mathrm{~kg}$ pcrPET flakes were used for hot washing with $\mathrm{NaOH}$ and FLO detergent dosage at elevated temperature.

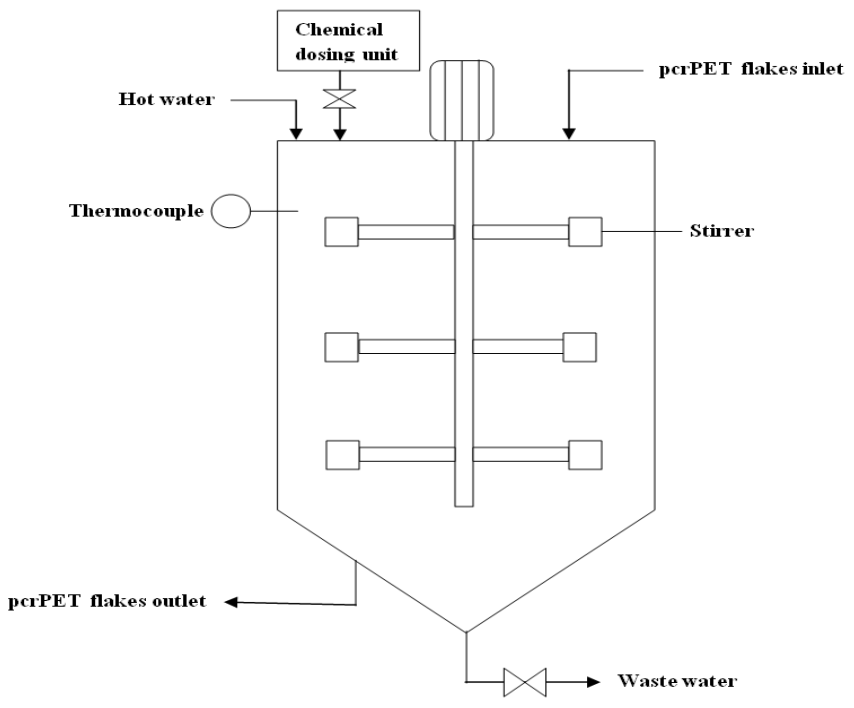

Fig. 1 Schematic of a hot washer.

\subsection{Sample Collection}

pcrPET bottles sample was collected from five big suppliers who collected bottles through their small collection hub across the country and $2 \mathrm{~kg}$ sample was collected from each supplier. Moreover, another $2 \mathrm{~kg}$ pcrPET bottles sample was collected directly from waste collection bins and landfills. Later, all the samples, i.e., $12 \mathrm{~kg}$ bottles were mixed together to homogenize pcrPET bottles generated throughout the country. However, each time $0.1 \mathrm{~kg}$ pcrPET bottles were taken from homogenized sample for subsequent sample pretreatment.

\subsection{Sample Pretreatment}

pcrPET bottles taken from homogenized sample also contained other plastics such polyethylene (PE), polypropylene (PP), polyvinyl chloride (PVC) etc. and metals alongside pcrPET due to lack of proper post consumer plastic waste collection method in Bangladesh. The undesired plastics were removed manually in this step.

\subsection{Cold Washing}

After initial pretreatment, pcrPET sample were washed with cold water at ambient condition to remove dirt from the sample in a rector called cold washer. Cold washer was mainly a 2 L bucket containing a slow speed stirrer (50 rpm) which accommodated initially taken pcrPET sample. Cold washing provided relatively clean pcrPET bottles which later chipped into desired size in subsequent processing steps.

\subsection{Sample Cutting}

Cleaned PET bottles sample were chipped into 7-13 mm sized pcrPET flakes (Fig. 2) in accordance with the recycled PET flakes size of several large recycled PET flakes manufacturer from Asia to Europe [20]-[22]. Sample cutting was carried out without any kind of drying.

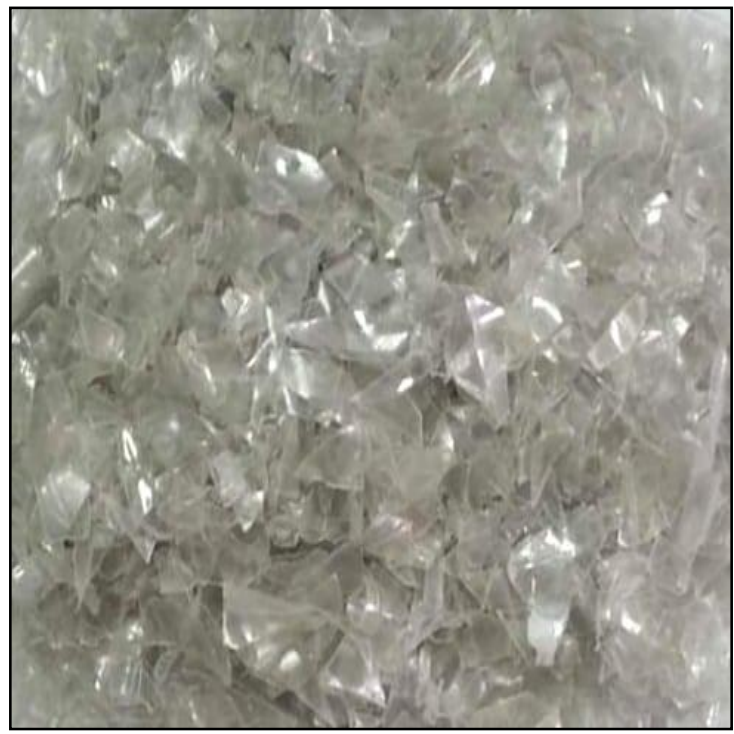

Fig. 2 pcrPET flakes sample for hot washing after cutting into desired $7-13 \mathrm{~mm}$ size.

\subsection{Gravity Separation}

Gravity separation in another water reactor was employed to separate PE, PP, PVC etc. materials from pcrPET flakes which finally provided flakes sample for hot washing. This flakes sample contained mostly glue and dirt contaminants which were removed in hot washing.

\subsection{Hot Washing}

Hot washing used $\mathrm{NaOH}$ and FLO detergent dose at $80{ }^{\circ} \mathrm{C}$ to remove contaminants from pcrPET sample flakes while lower and higher temperature was avoided due to ineffective washing and excessive foaming correspondingly. Hot washing was carried out in small scale reactor which has 30 minutes residence time for sample flakes. Moreover, this small reactor also contained a high speed stirrer $(100 \mathrm{rpm})$ for effective contact between flakes and hot washing chemicals. Chemical dose - $\mathrm{NaOH}$ and FLO detergent percentage - was changed gradually in both reactors until hot washing produced contaminants free quality recycled PET flakes. 
Actually, $\mathrm{NaOH}$ and FLO detergent dose percentages were employed as hot washer reactive volume (1 L). For example, detergent dose was slowly augmented from $1 \%$ to $3 \%$ percent of total hot washer volume (1 L) but $\mathrm{NaOH}$ dose was constant. Three different constant $\mathrm{NaOH}$ dose values such as 1, 2 and 3\% were selected. Each time different detergent dose, varying from $1 \%$ to $3 \%$, at a constant $\mathrm{NaOH}$ dose was used in hot washing. Then optimum chemical dose in hot washing reactor at $80^{\circ} \mathrm{C}$ temperature was determined from recycled PET flakes colorimetric analysis.

\subsection{Sample PET Flakes Washing and Drying}

After hot washing and before characterization by colorimetric analysis, recycled PET flakes were washed vigorously and dried. This is because the presence of trace amount of unused hot washing chemicals $\mathrm{NaOH}$ and FLO detergent) can produce yellowish color in recycled PET flakes. Therefore, extensive cold washing, for example, centrifugal washing, float washing and spray washing were carried out one after another for at least 10 minutes to remove unused hot washing chemicals. After hot washing chemicals removal, PET flakes were dried for 10 minutes at $130{ }^{\circ} \mathrm{C}$ for water removal. Drying of PET flakes produced contaminants free, dry and quality recycled PET flakes which were again dried at $220{ }^{\circ} \mathrm{C}$ for another half an hour.

\subsection{Recycled PET Flakes Colorimetric Analysis}

After consecutive dryings, recycled PET flakes from every chemical dose were stored individually. Stored recycled flakes from every single dose were used to prepare pcrPET solution with $0.025,0.05,0.1,0.15$, and 0.2 concentrations. Then a colorimeter was used for absorbance measurement against every concentration. Concentration vs. absorbance curve for different chemical doses were compared with pcrPET (without using any chemical dose) and pure PET to find out optimum chemical dose at hot washing. Before that two calibration curves, one for pure PET and another one for pcrPET with any chemical dose, were prepared for aforementioned comparative study.

\section{Results and Discussion}

\subsection{Calibration Curve of Pure PET and pcrPET}

Colorimeter absorbance was measured against different pure PET concentrations and results are represented in Fig. 3. Pure PET means those PET that have not used in any packaging or other applications. It is observed that absorbance is gradually increasing with the increment of PET concentrations. Results of absorbance at different concentrations of pcrPET are also shown in the same figure. Similar trend was observed in this case as like as that obtained in case of pure PET. However, higher absorbance value is achieved in case of pcrPET than pure PET as expected. Higher absorbance value is resulted from higher contaminations adherence after pcrPET bottles first hand use. Furthermore, every concentration of both types of PET is also compared. It was observed that for every single concentration, pure PET's absorbance is lower than that of pcrPET. This scenario was obtained since pure PET had no chance to adhere any kind of contaminations such as dirt, glue, PVC etc. as compared to pcrPET.

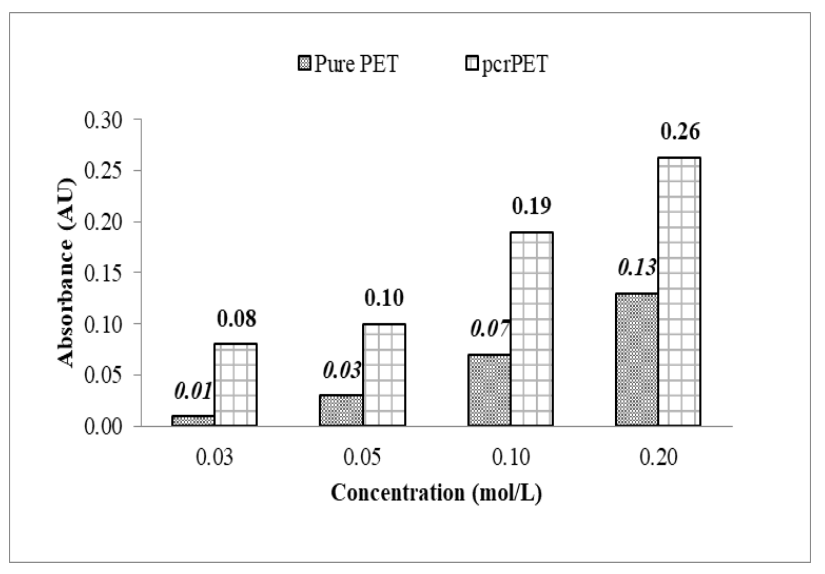

Fig. 3 Comparison of colorimeter absorbance between pure and pcrPET.

\subsection{Hot Washing with Varying Chemical Dose}

\subsubsection{Hot washing with $1 \% \mathrm{NaOH}$ and $1 \%, 2 \%$, and $3 \%$ FLO (detergent)}

Initially, PET flakes hot washing started with lower $\mathrm{NaOH}$ dose, $1 \%$, but detergent dose was increased gradually from $1 \%$ to $3 \%$. Among them lowest chemical dose, $1 \% \mathrm{NaOH}$ and $1 \%$ detergent, produced blackish recycled PET flakes (Fig. 4 a) after consequent drying at $130{ }^{\circ} \mathrm{C}$ and $220{ }^{\circ} \mathrm{C}$. $\mathrm{NaOH}$ used in the hot washing acted as aggressive cleaning agent for pcrPET flakes surface but such a lower dose $-1 \% \mathrm{NaOH}$ - could not remove mainly the dirt and glue contaminants from the flakes [8], [23]. As a result, discoloration of recycled PET flakes happened after consecutive drying since glue and dirt were identified as contaminants responsible for flakes discoloration by several authors [8], [24] However, blackish color from dirt contaminants offsets the slight yellowish color resulted from glue contaminants; resulted blackish recycled PET flakes at lower chemical dose [25]. Overall, as the lowest chemical dose did not remove contaminants such as dirt, glue etc. properly, colorimeter analysis of recycled PET flakes was not performed at this chemical dose.

Later, hot washing chemical dose was increased further to $1 \% \mathrm{NaOH}$ and $2 \%$ FLO level. Recycled PET flakes produced through this chemical dose are presented in Fig. 4 b whereas its colorimetric analysis is shown in Fig. 5. In Fig. 5, a comparative analysis between this recycled flakes with pure and pcrPET is drawn. Graphical representation at Fig. 5 shows that recycled flakes with this chemical dose stays in between the pure and pcrPET flakes. Actually, $1 \% \mathrm{NaOH}$ and $2 \%$ detergent dose (1\% vs $2 \%$ ) lowered the absorbance of recycled flakes highest $57.89 \%$ (at $0.01 \mathrm{~mol} / \mathrm{L}$ ) compared to pcrPET and on average this chemical dose lowered $40.25 \%$ absorbance. This implies that above chemical dose can significantly remove contaminations from pcrPET.

On the contrary, still there is a chance to improve contaminants removal as recycled PET flakes line stays above the pure PET line as shown in Fig. 5. Therefore, chemical dose again increased at $1 \% \mathrm{NaOH}$ and $3 \%$ FLO level. Although recycled flakes of this chemical dose were cleared satisfactorily, heavy foaming problem was encountered during PET flakes hot washing with this chemical dose. Therefore, 
colorimetric analysis was skipped for this chemical dose too since commercial application of this chemical dose is impractical due to heavy foaming at hot washing reactor.

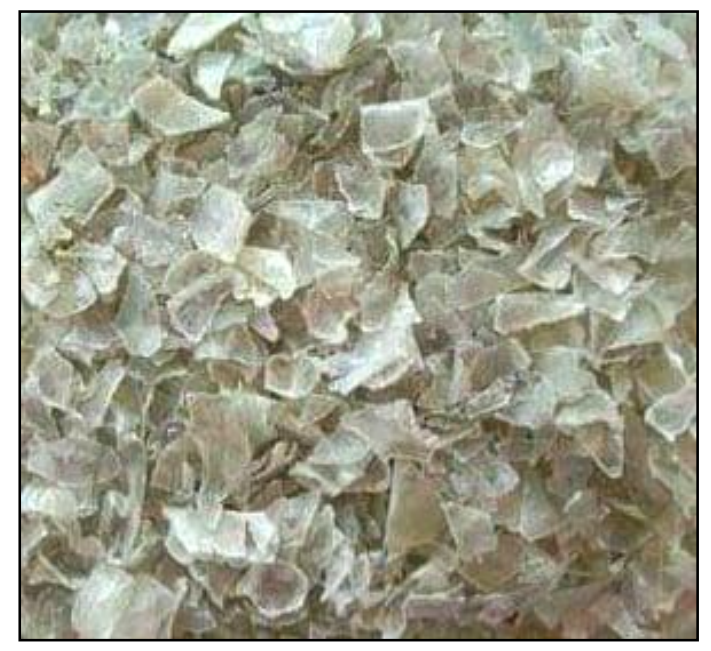

(a)

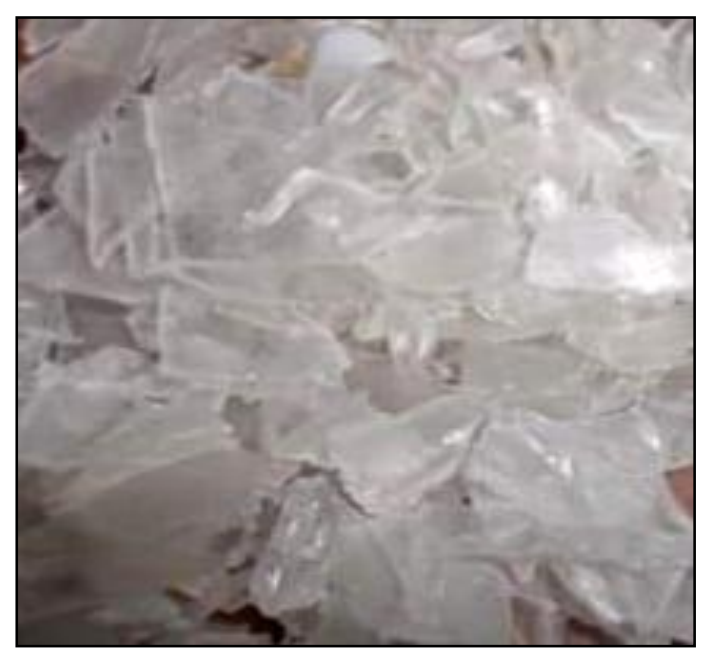

(b)

Fig. 4 Recycled PET flakes after hot washing and drying with (a) $1 \% \mathrm{NaOH}$ and $1 \%$ detergent (b) $1 \% \mathrm{NaOH}$ and $2 \%$ detergent.

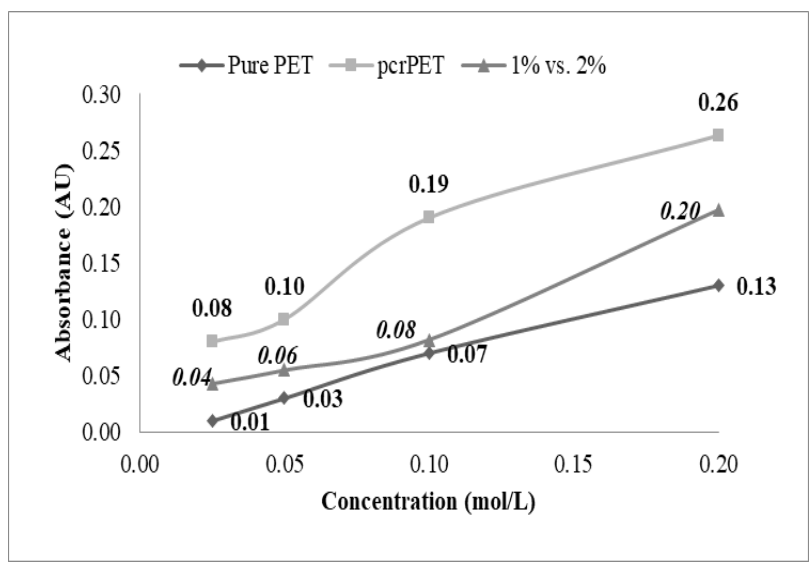

Fig. 5 Colorimetric analysis of recycled PET flakes with $1 \%$ $\mathrm{NaOH}$ and $2 \%$ percent FLO dose and comparative study between recycled and pure and pcrPET.
3.2.2 Hot washing with $2 \% \mathrm{NaOH}$ and $1 \%, 2 \%$, and $3 \%$ FLO (detergent)

As Fig. 5 suggests that further improvement is possible for contaminants removal, chemical dose was changed, especially, $\mathrm{NaOH}$ dose was increased to $2 \%$ and FLO dose was remained same as previous $1 \%, 2 \%$ and $3 \%$. However, lowest chemical dose in this combination again produced blackish recycled PET flakes and therefore colorimetric analysis was excluded for this dose.

Then subsequent chemical dose combination, $2 \% \mathrm{NaOH}$ and $2 \%$ FLO, produced comparatively cleaner recycled PET flakes and their colorimetric representation is shown in Fig. 6 (2\% vs. $2 \%$ ). Fig. 6 shows that highest $62.5 \%$ absorbance reduction (at $0.05 \mathrm{~mol} / \mathrm{L}$ concentration) is possible for recycled PET flakes with 2\% NaOH and 2\% FLO dose and on average $50.29 \%$ absorbance reduction is possible compared to pcrPET. This means that contaminants removal has been improved further due to chemical dose increase. As a result, recycled flakes line approaches further closer to pure PET line and away from pcrPET line in Fig. 6.

However, 2\% NaOH and 3\% FLO produces slightly yellowish recycled PET flakes instead of white and cleaned flakes after drying at $130{ }^{\circ} \mathrm{C}$ and $220{ }^{\circ} \mathrm{C}$. Moreover, huge foaming problem was also faced with this chemical dosage. Naturally detergents are prone to foam formation but in this chemical dose heavy foaming was formed due to addition of higher proportion of detergent to the very close to bubbling point of water during hot washing. Simultaneously large percentage of detergent and proximity of hot washing temperature $\left(80{ }^{\circ} \mathrm{C}\right)$ to bubbling point of water were responsible for heavy foaming [18], [26]. Due to above mentioned problems, commercial application of this chemical dose for pcrPET recycling is not suggested and the colorimetric analysis of this recycled PET is thus excluded.

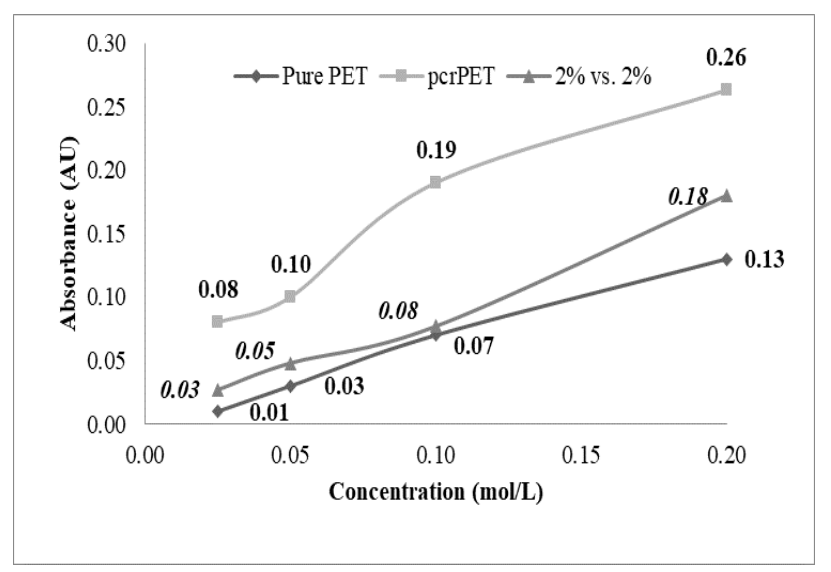

Fig. 6 Colorimetric analysis of recycled PET flakes with 2\% $\mathrm{NaOH}$ and $2 \%$ percent FLO dose and comparative study between recycled and pure and pcrPET.

3.2.3 Hot washing with $3 \% \mathrm{NaOH}$ and $1 \%, 2 \%$, and $3 \%$ FLO (detergent)

Previous hot washings were carried out at a constant $\mathrm{NaOH}$ dose either at $1 \%$ or $2 \%$ and varying percentages of FLO dose such $1 \%, 2 \%$ and $3 \%$. From previous result it was found that blackish recycled flakes were produced at lower chemical 
dose (1\% FLO) and foaming problem was encountered at higher chemical dose (3\% FLO). As a result, above two chemical doses were excluded while $\mathrm{NaOH}$ dose was increased further to $3 \%$.

However, $3 \% \mathrm{NaOH}$ and $2 \%$ percent FLO detergent produces recycled PET flakes which turned completely yellowish after subsequent drying at previously mentioned temperatures. This indicates that the extra addition of chemical during hot washing was not removed in several washing steps. As a result, remaining $\mathrm{NaOH}$ on PET flakes surface turned yellowish after successive drying at $130{ }^{\circ} \mathrm{C}$ and $220{ }^{\circ} \mathrm{C}$. This yellowish phenomenon can also be explained by natural tendency of $\mathrm{NaOH}$; turns yellowish after drying at elevated temperature [10], [19]. In addition, higher usage of $\mathrm{NaOH}$ incurs higher operating cost to PET recycling. As this chemical dose produces undesired recycled PET flakes, colorimetric analysis of this dosage has not also been included in this paper.

\subsection{Hot washing chemical dose optimization}

Recycled PET flakes produced through two best chemical doses (1\% NaOH and 2\% FLO, 2\% NaOH and 2\% FLO) at hot washing are compared together with pure and pcrPET in Fig. 7 to find out optimum chemical dose for hot washing. It can be observed that the absorbance of recycled flakes were always lower at every concentration compared to that of pcrPET. This means that the above two chemical doses are capable of removing attached contaminants from pcrPET. However, $2 \%$ $\mathrm{NaOH}$ and 2\% FLO dose has lower absorbance value than other chemical dose which implies that this chemical dose has higher efficiency for contaminants removal from pcrPET at every concentration. Moreover, the absorbance of recycled PET flakes corresponding to this chemical dose has closest proximity to pure PET, which clearly indicates that recycled PET flakes produced through this dose is closest to pure PETin terms of quality-than other recycled PET flakes. Therefore, $2 \% \mathrm{NaOH}$ and $2 \%$ FLO detergent dose is the best suited candidate for pcrPET flakes hot washing.

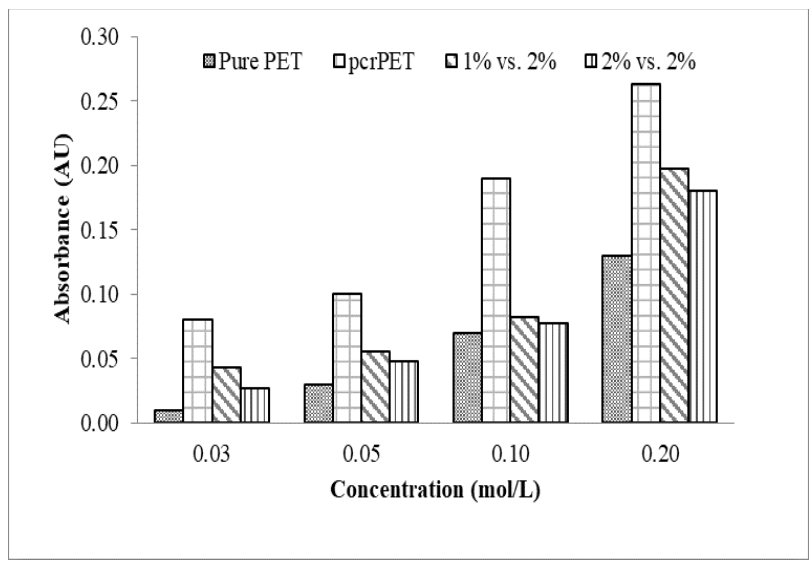

Fig. 7 Comparative study between pure and pcrPET with recycled PET at two chemical doses.

\section{Conclusion}

Mechanical recycling is an attractive process across the world for pcrPET bottles recycling due to its lower capital and operating cost involvement. Successful recycling mainly depends of contaminants removal from post consumer bottles after their first time use. This study used hot washing for effective contaminants removal. For hot washing, $\mathrm{NaOH}$ and detergent FLO was used in different proportions. Lower detergent dose, for example, $1 \%$ FLO, could not remove the dirt contaminants properly from the pcrPET resulted blackish recycled PET flakes. On the contrary, higher detergent dose 3\% FLO - created heavy foaming during the hot washing which made difficult to finish the hot washing and subsequent collection of recycled PET flakes for further colorimetric analysis. Similarly, higher $\mathrm{NaOH}$ dose such as $3 \% \mathrm{NaOH}$ produced yellowish recycled PET flakes (undesired quality) after hot washing and further drying. It occurred due to the presence of higher amount of $\mathrm{NaOH}$ at PET flakes surface after hot washing and later extensive cold washing. Finally, $1 \%$ $\mathrm{NaOH}$ and $2 \%$ detergent dose and $2 \% \mathrm{NaOH}$ and $2 \%$ detergent dose produced desired quality recycled PET flakes. Among them $2 \% \mathrm{NaOH}$ and $2 \%$ FLO detergent (a non ionic detergent) was found to be optimum chemical dose for hot washing since this chemical dose removed the highest percentage of contaminants from pcrPET. However, this paper studies only chemical dose optimization at the hot washing, but there are several other factors that should be considered for complete mechanical recycling of post consumer PET bottles.

\section{Acknowledgement}

This research was funded by SUST Research Centre in the year of $2017-18$.

\section{References}

[1] Bari, Q.H., Hassan, K.M. and Haque, M.E., 2012. Solid waste recycling in Rajshahi city of Bangladesh. Waste management, 32(11), pp.2029-2036.

[2] Matter, A., Ahsan, M., Marbach, M. and Zurbrügg, C., 2015. Impacts of policy and market incentives for solid waste recycling in Dhaka, Bangladesh. Waste Management, 39, pp.321-328.

[3] Mourshed, M., Masud, M.H., Rashid, F. and Joardder, M.U.H., 2017. Towards the effective plastic waste management in Bangladesh: a review. Environmental Science and Pollution Research,24(35), pp.2702127046.

[4] Rahman, S.M., Shams, S. and Mahmud, K., 2010. Study of solid waste management and its impact on climate change: a case study of Dhaka City in Bangladesh. In Proceedings of International Conference on Environmental Aspects of Bangladesh (ICEAB), September 4, 2010, University of Kitakyushu, Kitakyushu, Japan (pp. 229-231).

[5] Hasan, M.M., <IDLC Monthly Business Review - May 2015 .pdf >, in: C.R.M. Industry \& Equity Analysis Team, IDLC Finance Limited (Ed.) MONTHLY BUSINESS REVIEW, IDLC Finance Limited, Dhaka 1212, 2015, pp. $2-15$.

[6] U. Nations, U.N. Economic, S.C.f. Asia, t.P. Trade, I. Division, Enabling Environment for the Successful Integration of Small and Medium-sized Enterprises in 
Global Value Chains: Country Studies of Bangladesh, Nepal and Sri Lanka, United Nations ESCAP2012.

[7] Afroz, R., Hanaki, K. and Tudin, R., 2011. Factors affecting waste generation: a study in a waste management program in Dhaka City, Bangladesh. Environmental monitoring and assessment, 179(1-4), pp.509-519.

[8] Awaja, F. and Pavel, D., 2005. Recycling of PET. European Polymer Journal, 41(7), pp.1453-1477.

[9] Karayannidis, G.P., Nikolaidis, A.K., Sideridou, I.D., Bikiaris, D.N. and Achilias, D.S., 2006. Chemical recycling of PET by glycolysis: polymerization and characterization of the dimethacrylated glycolysate. Macromolecular Materials and Engineering, 291(11), pp.1338-1347.

[10] Malik, N., Kumar, P., Shrivastava, S. and Ghosh, S.B., 2017. An overview on PET waste recycling for application in packaging. International Journal of Plastics Technology, 21(1), pp.1-24.

[11] Nikles, D.E. and Farahat, M.S., 2005. New motivation for the depolymerization products derived from poly (ethylene terephthalate)(PET) waste: A review. Macromolecular Materials and Engineering, 290(1), pp.13-30.

[12] T.W.M. Organization, Best Practices in PET Recycling Clear PET, Clean Flake Specifications for Fiber, Bottles, Film and Sheet, Strapping, Engineered Resins, and Depolymerization, 1998.

[13] Park, S.H. and Kim, S.H., 2014. Poly (ethylene terephthalate) recycling for high value added textiles. Fashion and Textiles, 1(1), pp.1-17.

[14] Welle, F., 2011. Twenty years of PET bottle to bottle recycling - an overview. Resources, Conservation and Recycling, 55(11), pp.865-875.

[15] Yousuf, T.B. and Rahman, M., 2007. Monitoring quantity and characteristics of municipal solid waste in Dhaka City. Environmental monitoring and assessment, 135(13), pp.3-11.

[16] Sinha, V., Patel, M.R. and Patel, J.V., 2010. PET waste management by chemical recycling: a review. Journal of Polymers and the Environment, 18(1), pp.8-25.
[17] Romão, W., Franco, M.F., Bueno, M.I.M. and De Paoli, M.A., 2010. Distinguishing between virgin and postconsumption bottle-grade poly (ethylene terephthalate) using thermal properties. Polymer testing, 29(7), pp.879885.

[18] Mancini, S.D., Schwartzman, J.A.S., Nogueira, A.R., Kagohara, D.A. and Zanin, M., 2010. Additional steps in mechanical recyling of PET. Journal of Cleaner Production, 18(1), pp.92-100.

[19] Koo, H.J., Chang, G.S., Kim, S.H., Hahm, W.G. and Park, S.Y., 2013. Effects of recycling processes on physical, mechanical and degradation properties of PET yarns. Fibers and Polymers, 14(12), pp.2083-2087.

[20] E.-. recycling, Technical specifications: pet flakes quality specification, Poland, 2020. https://ergisrecycling.eu/en/content/PET-Polyethylene-

terephthalate/technical-specifications [Accessed 06 September 2012]

[21] M.R. Technology, PET Specification: Clear PET flakes, United Kingdom, 2020. http://marsrecyclingtechnology.co.uk/home/petspecification/_ [Accessed 06 September 2012]

[22] G.I. Limited, PET Bottle Flakes: Recycled PET Flakes, India, 2020. https://www.gravitaindia.com/pet-bottleflakes/ [Accessed 06 September 2012]

[23] Welle, F., 2016. Investigation into cross-contamination during cleaning efficiency testing in PET recycling. Resources, Conservation and Recycling, 112, pp.65-72.

[24] Welle, F., 2011. Twenty years of PET bottle to bottle recycling - an overview. Resources, Conservation and Recycling, 55(11), pp.865-875.

[25] Zhu, B., Liu, J., Pan, R., Wang, S. and Gao, W., 2015. Fabric seam detection based on wavelet transform and CIELAB color space: A comparison. Optik, 126(24), pp.5650-5655.

[26] Hossain, M.S. and Mozumder, S.I., 2018. Post consumer polyethylene terephthalate (PET) recycling in bangladesh through optimization of hot washing parameters. American Scientific Research Journal for Engineering, Technology, and Sciences (ASRJETS), 40(1), pp.62-76. 